\title{
Diskussion über Probleme der Geschichtsschreibung der Sozialdemokratie
}

\author{
Dieter Fricke
}

Bevor ich in Ergānzung bzw. gedanklicher Weiterführung meines Ihnen im Manuskript vorliegenden Referats auf einige methodologische Probleme eingehe, möchte ich zunāchst an die Diskussion von heute anknüpfen, in der die interessante Frage aufgeworfen worden ist, wo die Ursachen dafür liegen, daß sich in bestimmten Regionen bzw. Orten Linke oder auch Rechte stärker konzentrierten. Was den in der Diskussion mehrfach verwendeten Begriff der „Flügel“ betrifft, wird Ihnen bekannt sein, daß wir von politisch-ideologischen Strömungen sprechen. Strömungen, weil vieles ineinander fließt und keine klaren Abgrenzungen vorhanden waren. Wir vermeiden auch den Begriff Gruppe, weil es vor dem Ersten Weltkrieg, wenn wir von ganz wenigen Ausnahmen absehen, nichts Organisiertes in dieser Beziehung gab.

In besonderem Maße interessiert uns, wo Zentren der deutschen Linken waren und wie sie zustandekamen; etwa Bremen, Hamburg, Berlin, Leipzig, Stuttgart, Göppingen, aber auch Jena, um ein letztes Beispiel zu bringen. Jena, das immerhin mit dem Zeiss-Werk und Ernst Abbe besonders viele Arbeiter mit einem hohen Anteil an geistiger Arbeit hatte und wo auch der Grad der Arbeiteraristokratie relativ hoch war. Dennoch war Jena ein Zentrum der Linken. Nicht wenige Arbeiter nutzten hier die ihnen zur Verfügung stehende freie Zeit - in den Zeiss-Werken wurde schon um die Jahrhundertwende der Acht-Stunden-Arbeitstag eingeführt -, um sich mit dem wissenschaftlichen Sozialismus bzw. mit der Parteiliteratur zu beschäftigen. Es ist auch nicht zufällig, daß Jena Tagungsort der Osterkonferenz 1916 war, die einen entscheidenden Einschnitt in der Entwicklung der Arbeiterjugendbewegung bildete.

Damit komme ich eigentlich schon zu einer ersten These: Ich bin der Auffassung, daß es notwendig ist, die Komplexität der mit unserem Thema verbundenen Prozesse und auch die Frage nach den methodologischen Ansätzen ihrer Erforschung und Darstellung stärker zu berücksichtigen. Für einzelne solcher Prozesse und Erscheinungen kann das natürlich ein sozialgeschichtlicher oder ein wahlhistorischer oder auch ein alltagsgeschichtlicher Ansatz sein. Alles, wie ich betonen möchte, wichtige, unerläßliche, ja ehrenwerte Ansātze. Aber von dem Augenblick an, wo sie verabsolutiert, gewissermaßen für sakrosankt erklärt und die anderen Ansätze ignoriert werden, wird es nicht möglich sein, solche Probleme, wie ich sie hier andeutete, gründlich und umfassend zu untersuchen.

Meinerseits habe ich entsprechend meinem Verständnis vom Charakter der Arbeiterbewegung und ihrer Zielsetzung eine politische Geschichte der Arbeiterbewegung 
vor Augen, allerdings nicht eine deskriptive, oberflächliche, sondern unter Berücksichtigung solcher Ansätze, wie ich sie nannte, eine politische Geschichte, in der Politik nicht in konservativem Sinne verstanden wird, sondern in Richtung auf die Gedanken, die hierzu Friedrich Engels in seinen Altersbriefen dargelegt hat.

In bezug auf mein Thema gehört damit die Frage nach den bewußtseinsmäßigen Prozessen in der Arbeiterbewegung zu den vordringlichen; das heißt eigentlich mehr die nach dem subjektiven Faktor. Ich halte sie nicht zuletzt für wichtig, weil ich der Auffassung bin - und ich nehme an, sie werden mit mir übereinstimmen -, daß der politisch-ideologische Konstituierungsprozeß der Arbeiterklasse nicht mit den sechziger Jahren des 19. Jahrhunderts abgeschlossen ist, sondern weitergeht; und zwar sowohl in bezug auf die fortgeschrittenen Arbeiter, als auch hinsichtlich seiner Breite.

Was eine Definition des subjektiven Faktors anbelangt, schließe ich mich der Auffassung an, daß unter diesem nicht das Wirken einer Persönlichkeit, einer Klasse oder einer Partei schlechthin zu verstehen ist. Sein Wesen besteht vielmehr in einem auf die Veränderung oder Entwicklung der objektiven Bedingungen ausgerichteten Wirken, in der bewußten Tätigkeit der Werktätigen und ihrer Organisationen im historischen Prozeß. In der Arbeiterbewegung stellt der subjektive Faktor einen bestimmten Grad der Bewußtheit, des Willens, der Organisiertheit und der politischen Reife der Arbeiterklasse dar. Seinen Inhalt bilden der ideologische, der sozialpsychische, der politische, der moralische und andere Bereiche des Bewußtseins. Dabei fasse ich den subjektiven Faktor nicht als Ding an sich, sondern in seinen Wechselwirkungen mit den objektiven Bedingungen auf. Durch deren Reifegrad - in erster Linie durch den der ökonomischen Verhältnisse - wird er in seiner Aktivität bedingt, ohne aber von ihnen direkt abgeleitet zu sein, und auf sie wirkt er wieder zurück.

Diese Subjekt-Objekt-Dialektik spiegelt sich in der Geschichte der Arbeiterbewegung besonders in dem Wechselverhāltnis zwischen Partei, Klasse, Masse wider.

Davon abgeleitet bzw. damit korrespondierend geht es um das Verhältnis von Spontaneität und Bewußtheit in der Arbeiterbewegung, das sich historisch entwickelt und dessen einzelne, sehr unterschiedliche Entwicklungsstufen wiederum in jeder Phase der Arbeiterbewegung vorhanden sein können. Das betrifft auch das Phänomen der elementaren Arbeiterbewegung.

Damit hängt wiederum die Frage nach der Herausbildung des proletarischen Klassenbewußtseins zusammen; und zwar seiner einzelnen Entwicklungsstufen und Elemente ebenso wie von deren Verflechtung und gegenseitiger Abhängigkeit.

Und schließlich resultiert daraus ein besonders komplizierter Problemkomplex, nämlich die soziale Psyche der Arbeiterklasse. Ich verstehe darunter die Gesamtheit der für die Hauptmasse der Arbeiter typischen Stimmungen und Vorstellungen, Lebensnormen und Ideale, Bestrebungen und Bedürfnisse, die ihr gesellschaftliches Verhalten und den Grad ihrer Aktivität im Klassenkampf bestimmen, wobei spontane, emotionelle Reaktionen der Massen auf die gesellschaftliche Realitāt eine große Rolle spielen.

Daß das weder in einem psychologisierenden noch anthropologischen Sinne gemeint ist, brauche ich wohl nicht weiter darzulegen.

Wenn ich bei einem Colloquium über die Entwicklung der sozialistischen Arbeiter- 
bewegung zu einer Massenbewegung vor allem auf solche Fragen hinweise, dann erfolgt das in der Auffassung, daß die ganze Stärke der Arbeiterbewegung in entscheidendem Maße vom Grad ihrer Bewußtheit und ihres Massencharakters abhängt.

In diesem Zusammenhang halte ich es für notwendig, eine Lanze für eine entsprechende Beachtung organisationsgeschichtlicher Darstellungen und Untersuchungen zu brechen. Besonders seit den siebziger Jahren, als die Geschichte der Arbeiterbewegung von unten in der Bundesrepublik en vogue war, konnte man immer wieder Ressentiments gegen Ideologie- und aber auch gegen Organisationsgeschichte feststellen. Ressentiments, die ich nur für berechtigt halte, wenn etwa die Organisation formal und ohne deren blutvollen Inhalt aufgefaßt wird und auch nicht als ein „Drama von Ideen“. Oder wenn nur die organisierten, fortgeschrittensten Arbeiter gesehen werden und alle anderen Teile der Arbeiterklasse aus derm Blick geraten. Oder wenn es nur darum geht, das zu analysieren, was ich als „direktive“ Beschlüsse und Dokumente bezeichnen möchte, und nicht zu beachten, wie diese Beschlüsse in die Tat umgesetzt wurden und was aus ihnen geworden ist. Und ich würde dazu auch - heute Mittag wies Herr Bleek am Beispiel München darauf hin - das Wechselverhältnis zwischen der oberen und der unteren Parteiebene zählen.

Organisationsgeschichte steht für mich auch nicht im Widerspruch zur Individualitāt. Ich gehe davon aus, daß es eine Wechselwirkung zwischen Individuum, Klasse und Organisation gibt, und halte es nicht nur für möglich, sondern auch für notwendig, eine dritte methodologische Dimension einer wissenschaftlichen Analyse der Geschichte zu erschließen, nämlich Geschichte nicht nur als gesetzmäßigen, naturgeschichtlichen Prozeß und als Resultat menschlicher Tätigkeit, sondern auch als Entwicklung der menschlichen Individualitāt zu begreifen. Vorausgesetzt, daß dabei ein einheitliches methodologisches Prinzip der Erklārung aller geschichtlichen Erscheinungen beachtet wird, eröffnen sich damit für die Erforschung der Organisationsgeschichte und der Geschichte der Arbeiterbewegung überhaupt interessante Perspektiven.

Wenn ich solche Probleme nenne, dann möchte ich damit nicht die einleitend betonte Komplexität unseres Themas und das Erfordernis, die Geschichte der Arbeiterbewegung als eine politische Geschichte zu begreifen, in Frage stellen. Im Gegenteil, gerade dieser Ansatz ermöglicht es, auch bewußtseinsmäßigen, sozialpsychischen bzw. organisationsgeschichtlichen Aspekten so Rechnung zu tragen, daß sie weder unternoch überbewertet werden.

Damit komme ich zu einem Grundgedanken, der eigentlich selbstverstāndlich erscheint, gegen den aber meines Erachtens immer wieder verstoßen wird. Ich meine das auch von Herrn Ritter bereits gestern erwähnte Erfordernis, die Arbeiterbewegung nicht isoliert zu untersuchen. Ich fasse sie als einen systemimmanenten Bestandteil der bürgerlichen Gesellschaft auf und berücksichtige dabei besonders den dieser Gesellschaft innewohnenden und sie bestimmenden Widerspruch, der durch die Bourgeoisie und das Proletariat verkörpert wird und den Marx in bezug auf das Kapital und die Lohnarbeit als „zwei Seiten eines und desselben Verhāltnisses“ charakterisierte. Er schrieb weiter wörtlich: „Die eine bedingt die andere wie der Wucherer und Verschwender sich wechselseitig bedingen." 
Wenn wir uns in Jena seit Beginn der sechziger Jahre mit der Geschichte der bürgerlichen und kleinbürgerlichen Parteien und Verbände beschäftigt haben, dann geschah das vor allem aus der Überlegung heraus, daß die komplizierte Dialektik der Klassenauseinandersetzung zwischen Bourgeoisie und Proletariat ein eigenstāndiger, aber weitgehend nicht genügend beachteter Forschungsgegenstand ist und daß man die Geschichte der Arbeiterbewegung oder auch die Geschichte der Bourgeoisie nicht darstellen und analysieren kann, wenn man dieses Wechselverhältnis in seiner ganzen Kompliziertheit unbeachtet läßt.

Sollte ich es richtig verstanden haben, sind von Ihnen, Herr Ritter, hier in München ähnliche Ziele verfolgt worden. Und es freut mich, daß ich das gerade hier und heute bei allen grundsätzlichen und ja auch in den vergangenen Jahren nicht verschwiegenen sachlichen Differenzen im Herangehen an den Forschungsgegenstand und auch in den Forschungsergebnissen selbst aussprechen kann; und zwar nicht als Höflichkeitsfloskel gegenüber dem Gastgeber oder aus einer augenblicklichen Überschwenglichkeit heraus, sondern als eine ganz nüchterne, mir aber wichtig erscheinende und vielleicht auch ein wenig zukunftsträchtige Feststellung.

Eine Reihe von Gründen hat uns in Jena dazu veranlaßt, einen neuen Forschungsgegenstand zu wählen, der das Anliegen des bisherigen, also der Beschäftigung mit der Geschichte der bürgerlichen und kleinbürgerlichen Parteien und Verbände, auf einer neuen Stufe langfristig weiterführen soll. Erlauben Sie mir, daß ich das abschließend kurz skizziere, weil dieses Anliegen meines Erachtens in noch stärkerem Maße als unsere bisherigen Forschungen auch Fragestellungen dieses Kolloquiums nicht unwesentlich tangiert.

Es geht uns in Jena längerfristig um die Frage, wie die bürgerliche Gesellschaft in Deutschland seit 1789 politisch organisiert gewesen ist. Im Mittelpunkt unserer Forschungen soll die Gesamtheit der staatlichen und vorstaatlichen Institutionen und Organisationen stehen, in denen sich das politische Leben dieser Gesellschaft vollzog. Anders gesagt: Uns interessieren die materialisierten, institutionellen und organisatorischen Formen politischer Verhāltnisse.

Die politische Organisation der bürgerlichen Gesellschaft besteht für uns aus zwei wesentlichen Strukturelementen: dem Herrschaftsmechanismus und dem Mechanismus des proletarischen Klassenwiderstandes.

In Jena interessiert uns hauptsächlich das erste Strukturelement, ohne daß wir damit jedoch die zwischen beiden vorhandenen Wechselbeziehungen und auch Auseinandersetzungen außer acht lassen wollen. Das beträfe für uns beispielsweise solche Fragen, um einen Gedanken Antonio Gramscis aus seinen Gefängnisheften anzuführen: Wie sah das Befestigungssystem aus, das die herrschende Klasse gegenüber der Arbeiterbewegung bzw. den unterdrückten Massen errichtete, dieser mächtige Komplex von Schützengräben und Befestigungen? Wie wirkten seine einzelnen Teile zusammen? Bisher werden in der Regel die Parteien, die Verbānde, die Kirche, die Schule oder die Vereine bzw. der Staat und seine einzelnen Institutionen gesondert untersucht. Unser hauptsāchliches Interesse ist dagegen unter den von mir angedeuteten Aspekten auf die Verflechtung und das Zusammenwirken der einzelnen Elemente gerichtet, wobei es natürlich auch um die Entwicklung dieser Prozesse geht. 
Bei der schrittweisen Untersuchung dieses Systems, für das es bisher noch keine umfassende Darstellung seiner Geschichte gibt, wollen wir unsere Historikerseele nicht an die Soziologen verkaufen. Natürlich benötigen wir gründliche Kenntnisse über die Anatomie der politischen Organisation der bürgerlichen Gesellschaft. Unser Hauptanliegen wird aber darin bestehen, deren Physiologie zu erforschen und darzustellen. Das betrifft auch die für den Historiker unerläßlichen und außerst wichtigen philosophischen oder politischen Kategorien. Wir berücksichtigen jedoch, daß sie in der Geschichte selbst nicht existieren und nur theoretische Konstrukte sind.

Insgesamt gesehen geht es uns also um eine Orientierung auf gesamtgesellschaftliche und epochenübergreifende Prozesse. Wir hoffen, daß es uns auf diesem Wege möglich sein wird, auch zu einer noch eingehenderen und umfassenderen Beantwortung mancher Fragen einen Beitrag zu leisten, wie sie auf diesem Kolloquium zur Diskussion stehen.

\section{Klaus Schönhoven}

Sie haben am Anfang plädiert für eine politische Geschichte der Arbeiterbewegung. Wenn ich Ihre Begrifflichkeit, die Sie gewählt haben - Masse, Klasse, Partei - in den westlichen Sprachgebrauch übersetze, dann wäre das bei uns: Lage, Verhalten bzw. Bewußtsein und Organisation. Könnte man die drei Begriffe, die wir verwenden, in Ihren Sprachgebrauch übertragen?

Was macht aber den Charakter des Politischen aus? Ist es, wenn ich Ihr schriftliches Referat heranziehen darf, eine Metakategorie? Denn da heißt es, das Wachstum der SPD sei „zunehmend von folgenschweren Widersprüchen begleitet" gewesen, die sich auch „in der Tendenz einer Verflachung der Partei“ geāußert hätten. Hier kommt bei Ihnen nach meiner Auffassung die Metakategorie hinein. Woran messen Sie eigentlich die „Tendenz einer Verflachung“ und woran messen Sie den „Grad der Bewußtheit" in der sozialdemokratischen Arbeiterbewegung?

\section{Gerbard A. Ritter}

Ihre einleitenden grundsātzlichen Bemerkungen, die ja weit über den Gegenstand Ihres schriftlichen Referats hinausgehen, haben mich außerordentlich interessiert, da ich darin einen Beweis für die starken Parallelen in der Entwicklung der Forschung zur Arbeiterschaft und Arbeiterbewegung in der DDR und in der Bundesrepublik während der letzten Jahre sehe. Gleichzeitig finde ich frappierend, daß offenbar viele Forscher in der Bundesrepublik, zu denen ich auch mich rechnen möchte, inzwischen dazu tendieren, die Bedeutung der sozio-ökonomischen Grundbedingungen für die Entstehung und Entwicklung von Arbeiterschaft und Arbeiterbewegung eher stärker zu betonen als die von Ihnen repräsentierte Forschungsrichtung in der DDR. Allerdings hat auch für uns die politische Struktur - Sie nennen es Anatomie und Physiologie der politischen Organisation der bürgerlichen Gesellschaft - eine eigenständige 